\section{Associação entre o estresse oxidativo e estado inflamatório com variáveis extrapulmonares em tabagistas}

\section{Association between oxidative stress and inflammatory status with extrapulmonary variables in smokers}

\author{
Helenize Veron Lopes, Andressa Brum Félix, André Felipe Santos da Silva, Elizabeth do Canto \\ Brancher, Daniele Prestes, Eduardo Matias dos Santos Steidl, Renata Mancopes, Adriane \\ Schmidt Pasqualoto
}

\section{RESUMO}

Objetivo: Avaliar o estresse oxidativo e estado inflamatório de tabagistas e não tabagistas e analisar a associação com variáveis extrapulmonares. Métodos: Estudo transversal e descritivo, onde a amostra foi composta por 10 tabagistas (GT) e 10 não tabagistas. Após a espirometria, foram submetidos à análise do Hemograma, da Proteína C-Reativa us e TBARS e avaliação da força muscular respiratória, capacidade funcional e da qualidade de vida. A homogeneidade das variáveis foi testada pelo teste Levene, as diferenças entre os grupos foram analisadas pelo teste t de Student ou teste $U$ de MannWhitney. Associações entre as variáveis foram avaliadas pelo teste de correlação de Pearson ou Spearman. Resultados: GT apresentou carga tabágica de 29,49 \$33,66 anos/maço e consumo diário de cigarros de $13,5 \pm 8,5$. Foi encontrada diferença entre os grupos no nível de leucócitos $9750 \pm 2269$ $X 7320 \pm 1692(p=0,028)$ entre os grupos. Houve associação entre PCR-us e carga tabágica $(r=0,806$; $p=0,007)$ e entre TBARS e PEmáx $(r=0,818 ; p=0,006)$. Conclusão: houve presença de leucocitose no GT, bem como associação entre PCR-us e carga tabágica e entre PEmáx e TBARS. Não foram encontradas associações das demais variáveis com os níveis de TBARS e PCR-us.

Descritores: Fisioterapia, Hábito de Fumar, Estresse oxidativo.

\section{ABSTRACT}

Objective: To evaluate oxidative stress and inflammatory status of smokers and nonsmokers and analyze the association with extrapulmonary variables. Methods: Cross-sectional, descriptive study where the sample was composed of 10 smokers and 10 nonsmokers. After spirometry, they were subjected to analysis of blood count, C-reactive protein us and TBARS and evaluation of respiratory muscle strength, functional capacity and quality of life. The homogeneity of variances was tested by Levene test, the differences between the groups were analyzed by Student $t$ test or Mann-Whitney $\mathrm{U}$ test. Associations between variables were assessed by Pearson or Spearman correlation test. Results: GT introduced tobacco intake of 29,49 $\pm 33,66$ years/pack and daily cigarette consumption of $13,5 \pm 8,5$. Difference was found between FEV1 post in groups $2,30 \pm 0,59$ and $2,86 \pm 0,50(p=0,033)$, FEV1/FVC post 75,07 $\pm 12,62 \times 85,90 \pm 4,98$ ( $p=0,027)$ and leukocytes $9750 \pm 2269 \times 7320 \pm 1692$ $(p=0,028)$, respectively GT and GC. There was an association between PCR-us and smoking history $(p=0,007 ; r=0,806)$ and between TBARS and PEmáx $(p=0,006 ; r=0,818)$, both in GT. Conclusion: Only spirometry and leukocytosis levels were evident in the differences between groups. In the GT was no association between PCR-us and smoking history and between PEmáx and TBARS. There were no associations of other variables with the levels of TBARS and PCR-us.

Descriptors: Physiotherapy, Tobacco, Oxidative stress. 


\section{INTRODUÇÃO}

O tabagismo tornou-se uma epidemia mundial, representando uma grande ameaça à saúde pública e causando a morte de cerca de seis milhões de pessoas por ano. Dentre estas mortes, 600.000 são decorrentes do fumo passivo e mais de cinco milhões ocorrem pelo uso direto do tabaco, podendo aumentar para mais de oito milhões até $2030^{1}$.

A fumaça do cigarro contém mais de 4.000 substâncias químicas, sendo componentes tóxicos, mutagênicos e carcinogênicos, causadores de doenças cardiovasculares e doenças pulmonares obstrutivas crônicas (DPOC)2. Embora os esforços para reduzir a prevalência do tabagismo tenham que ser reforçados, a compreensão dos processos que contribuem para o início e progressão de doenças relacionadas ao fumo é de igual importância, dada a natureza altamente viciante do cigarro ${ }^{3}$.

Estudos têm relatado que fumantes sem diagnóstico espirométrico de obstrução ao fluxo aéreo já apresentam níveis reduzidos de atividade física, capacidade funcional, função pulmonar, força dos músculos respiratórios, qualidade de vida, além de ansiedade e depressão, em relação a não fumantes. Esses resultados confirmam os diferentes aspectos do comprometimento da saúde entre os tabagistas ${ }^{4,5}$.

Além disto, a fumaça do cigarro está associada ao estresse oxidativo em diversos órgãos, pois contém elevadas concentrações de radicais livres e espécies reativas de oxigênio (EROS) ${ }^{3}$. As vias e mecanismos envolvidos na fisiopatologia de doenças associadas ao tabagismo estão relacionados em grande parte à lesão tecidual causada pelas EROS e por uma série de reações inflamatórias, que desencadeiam apoptose celular ${ }^{6-8}$. Essas vias muitas vezes interagem entre si, produzindo ciclos viciosos de estresse oxidativo e inflamação, contribuindo para a progressão da doença ${ }^{9}$. Existem várias linhas de evidência que sugerem que existe um desequilíbrio entre oxidantes e antioxidantes nos pulmões e no sangue de tabagistas ${ }^{10}$.

Diante do exposto, avaliar o nível de estresse oxidativo torna-se relevante na medida de esclarecer esta via de lesão e sua relação com os mecanismos inflamatórios. O presente estudo teve como objetivo comparar o estresse oxidativo e estado inflamatório de tabagistas e não tabagistas, e analisar a associação com variáveis de força muscular respiratória, capacidade funcional e qualidade de vida.

\section{MÉTODO}

Estudo transversal aprovado pelo Comitê de Ética em Pesquisa (CEP) da Universidade Federal de Santa Maria (UFSM) (protocolo $\left.n^{0} 13022213.1 .0000 .5346\right)$. Todos os participantes assinaram o termo de consentimento livre esclarecido antes da inclusão.

A amostra foi selecionada por conveniência e composta por 20 voluntários, com idades entre 30 e 70 anos, sendo 10 tabagistas ativos (Grupo Tabagista - GT) com consumo maior que 10 maços/ano, sem doença pulmonar crônica comprovado pelo laudo do exame espirométrico, e 10 não tabagistas (Grupo Controle - GC). Como critérios de exclusão foram adotados: portadores de doenças neurológicas, ortopédicas e autoimunes que pudessem interferir na capacidade de realização dos testes e na análise do perfil inflamatório.

\section{Procedimentos}

Inicialmente foi realizada a prova de função pulmonar (espirometria). Em ambos os grupos foi realizada anamnese e no GT, para coleta da história do tabagismo, foi aplicado o questionário de Menezes et al. ${ }^{11}$ adaptado. 
Na sequência, foram coletadas amostras sanguíneas para análise do Hemograma, Proteína C-Reativa ultra sensível (PCR-us) e substâncias reativas ao ácido tiobarbituríco (TBARS).

Após, foi realizado o teste de força muscular respiratória através da manovacuometria onde foram medidas a Pressão Inspiratória máxima (PImáx) e a Pressão Expiratória máxima (PEmáx), a avaliação da capacidade funcional pelo Teste do Degrau de 6 minutos (TD6'), para avaliação da qualidade de vida foi aplicado o questionário 12-Item ShortForm Health Survey (SF12) e para o nível de atividade física foi utilizado o Questionário Internacional de Atividade Física (IPAQ).

Espirometria

Todos os pacientes foram submetidos à avaliação espirométrica, em ambiente climatizado, utilizando-se do aparelho Espirômetro JAEGER MS IOS Digital. O teste consistiu de manobras de inspiração e expiração máximas, que foram realizadas no aparelho, até que se pudessem registrar três manobras reprodutíveis de acordo com as recomendações da SBPT - Sociedade Brasileira de Pneumologia e Tisiologia ${ }^{12}$. Os valores obtidos foram de acordo com Pereira et al. ${ }^{13}$.

Coleta sanguínea

Foram coletados $3 \mathrm{~mL}$ de sangue venoso através de punção da veia antecubital para análise do Hemograma e da PCR-us, e $5 \mathrm{~mL}$ de sangue venoso para análise do estresse oxidativo, por profissional habilitado. 0 método de ensaio da PCR-us foi baseado na turbidimetria com intensificação da reação por partículas de látex revestidas com anticorpos monoclonais anti-PCR. O precipitado foi determinado pelo método turbidimétrico a 540nm. Para análise do estresse oxidativo o sangue foi centrifugado durante $5 \mathrm{~min}$ a 3000 rotações por minuto (rpm). Após, separado o plasma, o pellet de glóbulos vermelhos foi lavado três vezes com igual volume de solução fisiológica, em seguida descartado o sobrenadante e reservado $100 \mu \mathrm{L}$ dos glóbulos vermelhos para medida do TBARS ${ }^{14}$.

\section{Manovacuometria}

A força muscular respiratória foi mensurada através das PImáx e PEmáx, por meio do manovacuômetro modelo digital MVD300®, Microhard System, Globalmed, Porto Alegre, RS, Brasil.

Com o indivíduo sentado foram realizadas cinco mensurações de cada pressão, com duração de no mínimo 1 segundo, com um minuto de intervalo entre cada uma. Para validação, deveriam ocorrer pelo menos três manobras reprodutíveis (uma com variação igual ou inferior a 10\% e a outra com variação de, no máximo, 20\% com a pressão de maior valor), sendo a de maior valor adotada no estudo como força muscular respiratória do indivíduo ${ }^{15}$.

Os valores de referência utilizados foram de acordo com o previsto por Neder et al. ${ }^{16}$.

TD6'

O TD6', que objetiva a avaliação da capacidade funcional dos participantes, foi realizado utilizando um degrau único de $20 \mathrm{~cm}$ de altura, com plataforma emborrachada. As recomendações e contraindicações, cuidados com a 
segurança do participante e critérios para interrupção do TD6' são os mesmos recomendados para o teste de caminhada de seis minutos ${ }^{17}$.

Foi realizado incentivo durante a realização do teste, preconizando estímulos padronizados, onde a cada minuto foi informado o tempo restante do teste para o participante. Durante todo o teste, foram monitorados dados referentes à frequência cardíaca $(\mathrm{FC})$, saturação periférica de oxigênio $\left(\mathrm{SpO}_{2}\right)$, pressão arterial $(\mathrm{PA})$, frequência respiratória $(\mathrm{FR})$, sensação de dispneia e fadiga de membros inferiores (MMII) pela escala de Borg modificada ${ }^{18}$. O número de degraus subidos com os 2 pés foi registrado no final dos seis minutos.

\section{SF-12}

O SF-12 é um questionário composto por doze itens, os quais avaliam os domínios mental (Mental Health Score) e físico (Physical Health Score), na percepção da qualidade de vida em relação à saúde.

A pontuação obtida produz escores de $0-100$, onde os valores são divididos por cores, sendo que: vermelha (30 a 38) - indica que o indivíduo está bem abaixo da média da população geral na qualidade de vida; amarela (38 a 43) estando na média; verde (43 a 70) - estando na média ou acima da média da população geral na qualidade de vida ${ }^{19}$. As doze respostas geram uma pontuação para o domínio físico e para o domínio mental, possibilitando a adoção de pontos de corte satisfatórios para o nível de qualidade de vida, sendo 50 pontos para o domínio físico e 42 pontos para o domínio mental ${ }^{20}$.

O questionário foi aplicado sob a forma de entrevista, durante a qual as perguntas de cada domínio foram lidas, de maneira clara e compreensível, para os participantes respondê-las, sem haver influência na resposta.

Nível de atividade física

O nível de atividade física foi classificado de acordo com o IPAQ versão 8, na sua forma curta: insuficientemente ativo, suficientemente ativo e muito ativo ${ }^{21}$. 0 trabalho de campo foi realizado por duas entrevistadoras e dois auxiliares. A equipe foi submetida a um treinamento para aplicação do questionário e padronização das entrevistas.

\section{Análise estatística}

Foi realizada a estatística descritiva para representação dos grupos da pesquisa. Para a realização dos testes de hipóteses foi realizado inicialmente o teste de normalidade de Shapiro-Wilk e a verificação da homogeneidade das variâncias através do teste de Levene. Já para a análise das diferenças entre grupos nas variáveis simétricas foi utilizado a teste $t$ de Student e para as assimétricas foi utilizado o teste $U$ de Mann-Whitney.

Para as correlações das variáveis foi utilizado o teste de correlação de Pearson para as variáveis simétricas e 0 teste de correlação de Spearman para as assimétricas. O nível de significância adotado foi de $5 \%$ e o software utilizado foi o Statistical Package for the Social Sciences (SPSS 17.0) para Windows.

\section{RESULTADOS}

No total foram avaliados 20 sujeitos ( $G T=10$ e $G C=10)$. O GT foi constituído de quatro homens e seis mulheres, e o GC por três homens e sete mulheres $(p=0,648)$. Com relação à idade, peso, altura e IMC, não foram encontradas 
diferenças significativa entre os grupos. No que se refere à qualidade de vida, não houve diferença significativa entre os grupos, porém os sujeitos do GT apresentaram valores menores nos dois domínios (Tabela 1).

Tabela 1 - Medidas antropométricas, qualidade de vida e nível de atividade física dos indivíduos não tabagistas e tabagistas.

\begin{tabular}{lccc}
\hline Variáveis & GT & GC & p \\
\hline Idade (anos) & $51,30 \pm 8,26$ & $48,00 \pm 6,22$ & $0,326^{1}$ \\
Peso (kg) & $68,00 \pm 14,11$ & $70,80 \pm 15,66$ & $0,820^{2}$ \\
Altura (m) & $1,63 \pm 0,09$ & $1,67 \pm 0,10$ & $0,400^{1}$ \\
IMC (kg/m $)$ & $25,45 \pm 3,59$ & $25,42 \pm 4,44$ & $0,986^{1}$ \\
SF12 PCS & $49,39 \pm 7,72$ & $50,56 \pm 8,17$ & $0,449^{2}$ \\
SF12 MCS & $53,44 \pm 8,73$ & $54,29 \pm 8,94$ & $0,832^{1}$ \\
IPAQ (n/\%) & & & \\
\multicolumn{1}{c}{ Ativos $\quad$ Muito ativos } & $7 / 70 \%$ & $4 / 40 \%$ & $0,328^{3}$ \\
\hline
\end{tabular}

IMC: Índice de massa corpórea; SF 12 PCS: Questionário de qualidade de vida - Domínio Físico; SF 12 MCS: Questionário de qualidade de vida - Domínio Mental; IPAQ: Questionário Internacional de Atividade Física. ${ }^{1}$ Teste t de student. ${ }^{2}$ Teste U de Mann-Whitney. ${ }^{3}$ Teste Exato de Fisher.

Em relação ao histórico de tabagismo (GT), verificou-se que a média de cigarros fumados por dia foi de 13,5 $\pm 8,5$ e a média de idade em que começaram a fumar foi de $20 \pm 10$ anos. A carga tabágica foi de 29,49 333,66 maços/ano e apresentou uma correlação positiva com a PCR-us ( $r=0,806 ; p=0,007)$. Todos relataram que fumam cigarro industrializado.

As comparações entre grupos das variáveis de função respiratória e força muscular dos músculos respiratórios estão descritas na Tabela 2.

Tabela 2 - Comparação da função respiratória e força dos músculos respiratórios.

\begin{tabular}{|c|c|c|c|}
\hline Variáveis & GT & GC & $\mathrm{p}$ \\
\hline PIM $\left(\mathrm{cm} \mathrm{H}_{2} \mathrm{O}\right)$ & $79,30 \pm 22,55$ & $74,20 \pm 41,88$ & $0,684^{1}$ \\
\hline PI prevista $\left(\mathrm{cm} \mathrm{H}_{2} \mathrm{O}\right)$ & $96,71 \pm 15,49$ & $95,36 \pm 12,03$ & $0,570^{2}$ \\
\hline $\mathrm{PI} \%$ & $81,48 \pm 17,06$ & $76,15 \pm 26,45$ & $0,599^{1}$ \\
\hline PEM $\left(\mathrm{cmH}_{2} \mathrm{O}\right)$ & $101,00 \pm 23,52$ & $\begin{array}{l}97,80 \pm 32,22 \\
98,01 \pm 16,73\end{array}$ & $0,803^{1}$ \\
\hline PE prevista $\left(\mathrm{cmH}_{2} \mathrm{O}\right)$ & $99,90 \pm 20,59$ & & $0,570^{2}$ \\
\hline PE\% & $102,49 \pm 22,09$ & $98,56 \pm 22,15$ & $0,696^{1}$ \\
\hline TD6 & $126,50 \pm 28,49$ & $123,40 \pm 26,22$ & $0,803^{1}$ \\
\hline CVF predito (L) & $3,63 \pm 0,83$ & $3,51 \pm 0,78$ & $0,741^{1}$ \\
\hline
\end{tabular}




$\begin{array}{lccc}\text { CVF pós (L) } & 3,15 \pm 1,04 & 3,35 \pm 0,71 & 0,174^{1} \\ \text { VEF }_{1} \text { predito (L) } & 2,95 \pm 0,65 & 2,96 \pm 0,55 & 0,950^{1} \\ \text { VEF }_{1} \text { pós (L) } & 2,30 \pm 0,59 & 2,86 \pm 0,50 & 0,033^{1} \\ \text { VEF }_{1} \text { /CVF predito } & 80,93 \pm 1,94 & 84,72 \pm 711 & 0,162^{1} \\ \text { VEF }_{1} \text { /CVF pós } & 75,07 \pm 12,62 & 85,90 \pm 4,98 & 0,027^{1}\end{array}$

PIM: Pressão inspiratória máxima; PI: Pressão inspiratória; PEM: Pressão expiratória máxima; PE: Pressão espiratória; TD6': Teste do degrau de 6 minutos; CVF: Capacidade vital forçada; VEF1: Volume expiratório forçado no primeiro segundo; VEF1/CVF: Índice de tiffeneau. ${ }^{1}$ Teste t de student. ${ }^{2}$ Teste $\mathrm{U}$ de Mann-Whitney.

Foram encontradas diferenças significativas entre os grupos nas variáveis $\mathrm{VEF}_{1}$ pós e CVF/VEF 1 pós. Não houve diferenças significativas entre os grupos nas demais variáveis relacionadas à função pulmonar. Também não apresentaram diferenças significativas, entre os grupos, as variáveis relacionadas à força dos músculos respiratórios e ao TD6'.

Os resultados das análises do hemograma e de estresse oxidativo estão descritos na Tabela 3.

Tabela 3 - Hemograma e análise do estresse oxidativo em indivíduos não fumantes e fumantes.

\begin{tabular}{lccc}
\hline Variáveis & GT & GC & P \\
\hline & & 1 & $0,152^{1}$ \\
Hemoglobina (g/dl) & $13,72 \pm 0,7$ & $2,83 \pm 1,61$ & \\
& & & \\
Hematócrito (\%) & $41,6 \pm 2,84$ & $38,79 \pm 3,96$ & $0,153^{1}$ \\
Hemácias (milhões $/ \mathrm{mm}^{3}$ ) & $4,62 \pm 0,26$ & $4,49 \pm 0,47$ & $0,564^{1}$ \\
Leucócitos (células $/ \mathrm{mm}^{3}$ ) & $9750 \pm 2269$ & $7320 \pm 1692$ & $0,028^{1}$ \\
Bastões (células/mm $\left./ \mathrm{mm}^{3}\right)$ & $175,83 \pm 90$ & $151,80 \pm 94,70$ & $0,623^{1}$ \\
Segmentados (células/mm $/ \mathrm{mm}^{3}$ ) & $175,83 \pm 90,02$ & $151,80 \pm 94,07$ & $0,104^{2}$ \\
Eosinófilos (células $/ \mathrm{mm}^{3}$ ) & $223,5 \pm 102,79$ & $161,50 \pm 126,87$ & $0,129^{2}$ \\
Linfócitos (células $/ \mathrm{mm}^{3}$ ) & $3384,17 \pm 1242,86$ & $2303,10 \pm 510,88$ & $0,088^{1}$ \\
Monócitos (células $/ \mathrm{mm}^{3}$ ) & $682,17 \pm 404,90$ & $481,60 \pm 137,44$ & $0,193^{2}$ \\
Plaquetas (células $/ \mathrm{mm}^{3}$ ) & $225833 \pm 79908$ & $246300 \pm 60037$ & $0,568^{1}$ \\
TBARS & $0,52 \pm 0,44$ & $0,17 \pm 0,02$ & $0,070^{2}$ \\
PCR-us (mg/dl) & $0,52 \pm 0,44$ & $4,55 \pm 6,78$ & $0,597^{2}$ \\
\hline
\end{tabular}

TBARS: Peroxidação lípidica; PCR us: Proteína C Reativa ultra sensivel. ${ }^{1}$ Teste t de student. ${ }^{2}$ Teste U de Mann-Whitney.

Na comparação entre grupos, apenas a quantidade de leucócitos mostrou-se significativa $(p=0,028)$. Nos demais componentes do hemograma, assim como na análise do estresse oxidativo, não foram encontradas diferenças entre os grupos, todavia percebe-se uma tendência ao aumento dos níveis de TBARS dos tabagistas em relação aos não 
tabagistas.

Correlação dos níveis de TBARS com a Pemáx $(r=0,818 ; p=0,006)$ foi evidenciada no GT. Não foram encontradas associações do estresse oxidativo com as demais variáveis de força muscular respiratória, capacidade funcional e qualidade de vida em ambos os grupos.

\section{DISCUSSÃO}

O tabagismo é considerado uma pandemia silenciosa, uma vez que milhões de óbitos anuais são decorrentes de doenças relacionadas ao tabaco. 0 presente estudo objetivou avaliar o estresse oxidativo e estado inflamatório de tabagistas e não tabagistas e analisar a associação com variáveis de força muscular respiratória, capacidade funcional e qualidade de vida.

A resposta inflamatória induzida pelo estresse oxidativo e pelos componentes químicos do cigarro é observada pelo aumento do recrutamento de leucócitos no endotélio vascular e pelo aumento das concentrações de citocinas próinflamatórias, que além de aumentar a interação leucócito - célula endotelial, levam ao maior recrutamento de leucócitos que perpetuam o processo inflamatório ${ }^{8,22}$.

No presente estudo foi evidenciado aumento significativo na contagem de leucócitos no GT em comparação ao GC. Este achado corrobora com os resultados de Lakishmi et al. ${ }^{23}$ onde foram analisados 40 fumantes e 40 não fumantes com faixa etária entre 25 e 40 anos para avaliar o efeito do tabagismo e sua intensidade sobre os parâmetros hematológicos e lipídicos. Os autores encontraram um aumento estatisticamente significativo na contagem total de leucócitos, hematócrito e hemoglobina em fumantes. Com base no número de anos-maço, os fumantes foram classificados como leves (10-14), moderados (15-19), e pesados (acima de 20).

A quantificação de maços/ano fumados é importante no atendimento clínico, onde o grau de exposição ao tabaco está intimamente correlacionado com o risco de doenças. Comparando-se parâmetros hematológicos entre fumantes leves, moderados e pesados, o estudo mostrou que o número de hemácias, leucócitos totais e, em particular, os neutrófilos foram significativamente aumentados em fumantes pesados. $O$ aumento da contagem de leucócitos entre os fumantes pode ser devido à liberação de catecolaminas induzida pela nicotina. Inflamação na árvore brônquica devido ao efeito irritante da fumaça do cigarro pode ser um fator contributivo para a maior contagem de leucócitos totais entre os fumantes ${ }^{24}$.

Ainda, na amostra tabagista avaliada observa-se maiores valores de hematócrito e hemoglobina. A exposição excessiva de monóxido de carbono (CO) pode produzir policitemia em seres humanos, bem como em animais. A meiavida do $\mathrm{CO}$ no corpo é de três a cinco horas. Em uma pessoa que fume frequentemente e de forma contínua, os níveis de carboxi-hemoglobina aumentam e produzem uma hipoxemia progressiva, e conforme o CO liga-se com a hemoglobina, uma anemia funcional é produzida. Isto provoca má oxigenação dos tecidos e mudança nos parâmetros hematológicos ${ }^{25}$.

Em relação ao estresse oxidativo, avaliado pelas TBARS, não foi encontrada diferença significativa entre os grupos, no entanto, no GT, nota-se uma tendência ao maior desequilíbrio oxidativo ( $p=0,070)$. Estudos relataram aumento das TBARS no soro e no condensado do ar exalado de fumantes adultos, com médias de 10-17 cigarros fumados/dia por mais de 10 anos ${ }^{26,27}$. Porém, cabe salientar que a amostra destes estudos foram compostas por um maior número de indivíduos sem avaliação espirométrica e ainda, com maior número de cigarros fumados ao dia, podendo estes aspectos terem sido motivo para os diferentes achados em relação ao presente estudo.

Importante achado nos resultados foi a associação entre os níveis das TBARS e a PEmáx no GT. O estresse 
oxidativo e inflamação são mutuamente inter-relacionados. Considerando que o primeiro pode atuar como um sinal para a expressão de mediadores inflamatórios, este último pode modular o nível de produção de EROS e, portanto, do estresse oxidativo. Um número significativo de estudos investigaram as alterações metabólicas e estruturais que ocorrem nos músculos esqueléticos. A maior parte destes estudos foram realizados em músculos dos membros, dadas as dificuldades para ter acesso a amostras de músculos respiratórios ${ }^{28}$.

Danos na musculatura, decorrente do desequilíbrio oxidativo inflamação, ativação de vias proteolíticas, com degradação de proteínas, e até mesmo alguns sinais de miopatia, com falha na transmissão neuromuscular, foram descritos na musculatura periférica de pacientes com DPOC e podem pôr em risco a sua função, ocasionando descondicionamento, com consequente fraqueza da musculatura expiratória ${ }^{28}$.

A fim de avaliar os níveis de PCR-us em indivíduos saudáveis e tabagistas sem manifestações clínicas de DPOC, correlacionando-os com a função pulmonar e a carga tabágica, Weis et al. ${ }^{29}$ analisaram 30 indivíduos tabagistas e 30 não-tabagistas com idade entre 18 e 40 anos, de ambos os sexos. Não houve diferença estatisticamente significativa entre os valores de PCR-us nos fumantes e não fumantes, havendo apenas uma correlação significativa entre os níveis de PCR-us e a carga tabágica medida em maços/ano. Os tabagistas que fumavam mais de 10 maços/ano apresentaram níveis de PCR-us significativamente maiores em relação aos que fumavam menos de 10 maços/ano.

Em outro estudo, Pinto-Plata et al. ${ }^{30}$ recrutaram 88 pacientes com DPOC, com grau de obstrução de moderado a grave, 33 tabagistas e 38 não tabagistas, e realizou a análise da PCR-us, onde foi significativamente maior no grupo com DPOC. No entanto, não foi observada nenhuma diferença entre os grupos de fumantes e não fumantes. Da mesma forma, não houve diferença entre os pacientes com DPOC que eram fumantes atuais e aqueles que eram ex-fumantes. Segundo os autores, estes resultados podem ser devidos à falta de algum fator forte para determinar a diferença entre os dois grupos, a ausência de uma resposta inflamatória nos tabagistas, ou inflamação persistente em pacientes com DPOC mesmo após a cessação do tabagismo.

Os achados dos autores acima citados vão ao encontro dos resultados do presente estudo, onde não foi encontrada diferença significativa entre os valores de PCR-us no GT e GC. Entretanto, cabe ressaltar que houve uma correlação positiva entre a carga tabágica e os níveis de PCR-us no GT, alertando para possíveis alterações sistêmicas que já possam estar ocorrendo nestes sujeitos.

Em relação à qualidade de vida, não houve diferença significativa entre os grupos tanto no domínio mental quanto no domínio físico, porém os tabagistas apresentaram valores abaixo do ponto de corte satisfatório para o nível de qualidade de vida do domínio físico. Diferentemente dos resultados do presente estudo, Camelier ${ }^{19}$ coloca, quanto à exposição ao tabaco, que o domínio mental pareceu ser melhor marcador de piora na qualidade de vida, tendo uma média estatisticamente menor, aspecto não alcançado pelo domínio físico.

Com relação aos valores obtidos no TD6' também não houve diferença significativa entre o GT e GC. Embora este teste seja amplamente utilizado, estudos utilizando o teste do degrau em tabagistas são escassos, sendo grande parte com a utilização do teste de caminhada de seis minutos. Acredita-se que tanto os dados obtidos referentes às pressões respiratórias máximas e à capacidade funcional através do teste do degrau possam ser explicados pela atividade profissional exercida pelos tabagistas, pois apesar desta variável não ter sido avaliada, foi observado que a maioria dos tabagistas trabalhava em funções que exigem muita atividade, tais como, serviços gerais, serralheria, vidraçaria, entre outros.

Alguns aspectos referentes às limitações deste estudo podem ter influenciado nos resultados, a amostra foi composta por um número relativamente reduzido de indivíduos em cada grupo, selecionada por conveniência, o que 
não garante a representatividade da população. Pesquisas em indivíduos tabagistas devem ser realizadas com intuito de revelar outras evidências da relação do tabagismo com o estresse oxidativo e variáveis extrapulmonares.

\section{CONCLUSÃO}

Em conclusão, os resultados mostraram diferença nos níveis espirométricos e de leucocitose entre os grupos. Também evidenciou-se no GT a associação entre os níveis de PCR-us e a carga tabágica, e entre os níveis das TBARS com a PEmáx. $O$ estresse oxidativo, a força muscular respiratória, a capacidade funcional e a qualidade de vida não diferiram entre os grupos.

\section{REFERÊNCIAS BIBLIOGRÁFICAS}

1. World Health Organization. "Tobacco". 2015. [internet]. Brasil [acesso em 24 jun 2015]. Disponível em: http:/l www.who.int.

2. Alanazi A, Enezi FA, Alqahtani MM, Alshammari TF, Ansari MA, Al-Oraibi S et al. Effects of passive smoking on students at College of Applied Medical Sciences, King Saud Bin Abdulaziz University for Health Sciences, Riyadh. J Nat Sci Biol Med. 2015; 6(1):100-5.

3. Campos KKD, Manso RG, Gonçalves EG, Silva ME, Lima WG, Menezes CAS et al. Temporal analysis of oxidative effects on the pulmonary inflammatory response in mice exposed to cigarette smoke. Cell Immunol. 2013; 284:29-36.

4. Mesquita R, Gonçalves CG, Hayashi D, Costa VSP, Teixeira DC, Freitas ERFS et al. Smoking status and its relationship with exercise capacity, physical activity in daily life and quality of life in physically independent, elderly individuals. Physiotherapy. 2015; 101:55-61

5. Furlanetto KC, Mantoani LC, Bisca G, Morita AA, Zabatiero J, Proença M et al. Reduction of physical activity in daily life and its determinants in smokers without airflow obstruction. Respirology. 2014; 19:369-75.

6. Sangani RG, Ghio AJ. Lung injury after cigarette smoking is particle related. Int J Chron Obstruct Pulmon Dis. 2011; 6:191-198.

7. Mak JCW. Pathogenesis of COPD, Part II: Oxidative-antioxidative imbalance. The International Journal of Tuberculosis and Lung Disease. 2008;12(4):368-74.

8. Ambrose JA, Barua RS. The pathophysiology of cigarette smoking and cardiovascular disease an update. J Am Coll Cardiol. 2004; 43(10):1731-37.

9. Neves CDC. Efeito Crônico do Tabagismo no Perfil Inflamatório, Estresse Oxidativo e Desempenho Físico em Homens Assintomáticos. [Dissertação de Mestrado - Programa Multicêntrico de Pós-Graduação em Ciências Fisiológicas]. Diamantina (MG): Universidade Federal dos Vales do Jequitinhonha e Mucuri; 2014.

10. Nadeem A, Raj HG, Chhabra SK. Increased Oxidative Stress and Altered Levels of Antioxidants in Chronic Obstructive Pulmonary Disease. Inflammation. 2005; 29(1).

11. Menezes AMB, Victora CG, Hallal PC, Lisboa C, Torres C, Luna C et al. Platino: Projeto Latino-Americano de Investigação em Obstrução Pulmonar. 2002. [citado em 26 mar 2014]. Disponível em: <http://www.platino-alat. org>.

12. Sociedade Brasileira de Pneumologia e Tisiologia. Diretrizes para Testes de Função Pulmonar. J Bras Pneumol. 
2002; 28(3).

13. Pereira CAC, Barreto SP, Simões JG, Pereira FWL, Gerstler JG, Nakatani J. Valores de referência para espirometria em uma amostra da população brasileira adulta. J Pneumol. 1992; 18(1):10-22.

14. Oliveira G, Oliveira C, Dorado A, García-Fuentes E, Rubio E, Tinahones F et al. Cellular and plasma oxidative stress biomarkers are raised in adults with bronchiectasis. Clin Nutr. 2013; 32(1):112-7.

15. Pessoa IMBS, Neto MH, Montemezzo D, Silva LAM, de Andrade AD, Ferreira VF. Predictive equations for respiratory muscle strength according to international and Brazilian guidelines. Braz J Phys Ther. 2014; 18(5): 410-418.

16. Neder JA, Andreoni S, Lerario MC, Nery LE. Reference values for lung function tests. Maximal respiratory pressures and voluntary ventilation. Braz J Med Biol Res. 1999; 32(6):719-27.

17. Neder JA. Temas de revisão: TC6 e do degrau. Sociedade Brasileira de Pneumologia e Tisiologia. [periódico online]. 2007. [citado em: 08 abr 2015]. Disponível em: http://itarget.com.br/newclients/sbpt.org.br/2011/downloads/ arquivos/Revisoes/REVISAO_06_TESTE_CAMINHADA_DEGRAU.pdf.

18. Pasqualoto AS. Comparação das respostas fisiológicas no teste de exercício cardiopulmonar e em três testes de exercício submáximo em pacientes com doença pulmonar obstrutiva crônica. [Tese de Doutorado - Programa de Pós-Graduação em Ciências Pneumológicas]. Porto Alegre (RS): Universidade Federal do Rio Grande do Sul; 2009.

19. Camelier AA. Avaliação da qualidade de vida relacionada à saúde em pacientes com DPOC: Estudo de base populacional com o SF12 na cidade de São Paulo-SP. [Tese de Doutorado - Programa de Pós-Graduação em Medicina]. São Paulo (SP): Universidade Federal de São Paulo; 2004.

20. Ware JJ, Kosinski M, Keller SD. How to Score the SF-12 Physical and Mental Health Summary Scales. 2 ed. Boston, MA: The Health Institute, New England Medical Center. 1995.

21. Matsudo SM, Araújo T, Matsudo VR, Andrade D, Andrade E, Oliveira LC, et al. Questionário Internacional de Atividade Física (IPAQ): estudo de validade e reprodutibilidade no Brasil. Rev Bras Ativ Fís Saúde, 6:5-18, 2001.

22. Radin A, Cote C. Primary care of the patient with chronic obstructive pulmonary disease - Part 1: frontline prevention and early diagnosis. Am J Med. 2008; 21:S3-S12.

23. Lakshmi AS, Lakshmanan A, Kumar GP, Saravanan A. Effect of Intensity of Cigarette Smoking on Haematological and Lipid Parameters. J Clin Diagn Res. 2014; 8(7):BC11-BC13

24. Corre F, Lellouch J, Schwartz D. Smoking and leucocyte-counts. Results of an epidemiological survey. Lancet. $1971 ; 2: 632-34$

25. Tirlapur VJ, Gicheru K, Charalambous BM, Evans PJ, Mir MA. Packed cell volume, haemoglobin, and oxygen saturation changes in healthy smokers and non-smokers. Thorax. 1983; 38(10):785-87.

26. Aula FA, Qadir FA. Effects of cigarette smoking on some immunological and hematological parameters in male smokers in Erbil city. . Jordan J Biol Sci. 2013; 6(2):159-166.

27. Nowak D, Kalucka $S$, Białasiewicz $P$, Król M. Exhalation of $\mathrm{H}_{2} \mathrm{O}_{2}$ and thiobarbituric acid reactive substances (TBARS) by healthy subjects. Free Radic Biol Med. 2001; 30(2):178-186.

28. Gea J, Agusti A, Roca J. Pathophysiology of muscle dysfunction in COPD. J Appl Physiol. 2013; 114(9):12221234.

29. Weis L, Schwanck GB, Silva JS, Lenzi LGS, Machado MB, Balotin R, et al. O papel da Proteína C Reativa (PCR) na detecção precoce de inflamação sistêmica em fumantes. Revista da AMRIGS, Porto Alegre. 2007; 51(2):12831.

30. Pinto-Plata VM, Mullerova H, Toso JF. Feudjo-Tepie M, Soriano JB, Vessey RS, et al. C-reactive protein in patients 
with COPD, control smokers and non-smokers. Thorax. 2006; 61:23-8.

31. Danesh J, Wheeler JG, Hirschfield GM, Eda S, Eriksdottir G, Rumley A, et al. C- Reactive Protein and other circulating markers of inflammation in the prediction of coronary heart disease. N Engl J Med. 2004; 350(14):138797.

32. Agarwal R, Goldenberg M, Perry R, IsHak WW. The quality of life of adults with attention deficit hyperactivity disorder: a systematic review. Innov Clin Neurosci. 2012; 9:10-21.

33. Castro MG, Oliveira MS, Moraes JFD, Miguel AC, Araujo RB. Qualidade de vida e gravidade da dependência de tabaco. Rev. Psiq. Clín. 2007; 34(2):61-67.

34. Mitra M, Chung M, Wilber N, Walker D. Smoking status and quality of life. A longitudinal study among adults with disabilities. Am J Prev Med. 2004; 27(3):258-260.

35. Martinez JAB, Motas GA, Vianna ES, Oliveira JT. Impaired quality of life of healthy young smokers. Chest. 2004; 125(2):425-428. 